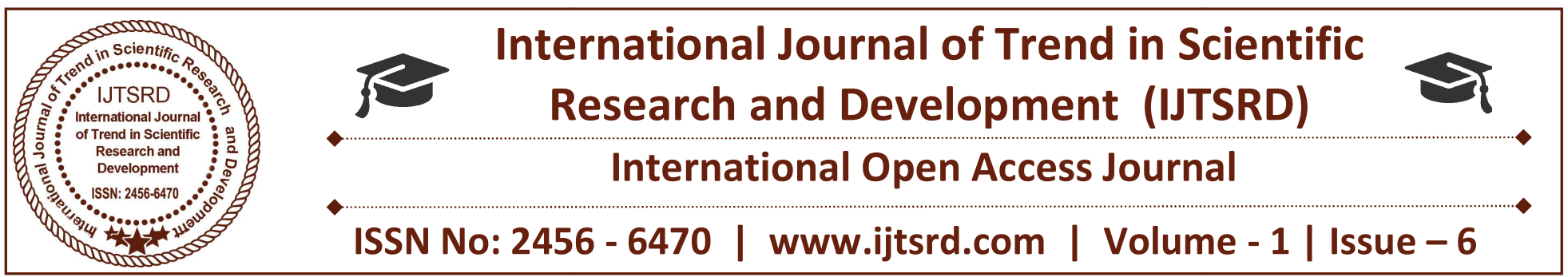

\title{
Radio-graphical Features of Gliosarcoma on Computed Tomography and Magnetic Resonance Imaging
}

\author{
Tamijeselvan. S. \\ Asst. Professor, Dept. of Radiography, \\ Mother Theresa Post Graduate and Research \\ Institute of Health Sciences, Puducherry, India
}

\section{ABSTRACT}

Gliosarcoma is a rare type of glioma, a cancer of the brain that comes from glial, or supportive, brain cells, as opposed to the neural brain cells. Gliosarcoma is a malignant cancer, and is defined as a glioblastoma consisting of gliomatous and sarcomatous components.

The aim of the study is to characterize the radiographic features of gliosarcoma, such as existence nature, content and appearance and enhancement on Computed Tomography and Magnetic Resonance Imaging. And also to find out which is the most effective imaging method to diagnose and categorize gliosarcoma.

Fifteen patients were studied retrospectively from the PACS system of CT scanner and MRI using the protocol of imaging of the head.

The study reveals that majority $(93.3 \%)$ of the gliosarcoma were single. About 80 percent of the gliosarcoma had both solid and cystic components. All fifteen tumors appeared to be relatively well demarcated from the surrounding normal or edematous brain parenchyma. On T2-weighted image eight shows of hyperintensity and isointensity.

The study concludes that Gadolinium enhanced Magnetic Resonance Imaging is more accurate than plain MRI, plain CT, Contrast Enhanced CT in defining the margins of Gliosarcoma.

Keywords: Gliosarcoma, Computed Tomography, Magnetic Resonance Imaging, Radiographical features

\section{INTRODUCTION}

Gliosarcomas are a variant of glioblastoma (along with epithelioid glioblastoma and giant cell glioblastoma) recognised in the current (2016) WHO classification of CNS tumours. They are highly malignant (WHO grade IV) primary intra-axial neoplasms with both glial and mesenchymal elements.

The vast majority of gliosarcomas arise from WHO grade IV astrocytomas (i.e. glioblastoma), however, rarely they can also arise from ependymomas or oligodendrogliomas in which case they can be referred to as ependymosarcomas and oligosarcomas respectivly.

Peak presentation is around the $6^{\text {th }}$ decade and there is a male predilection (M: F 1.8:1). Most tumours are primary but secondary tumours can occur in patients with previously resected glioblastomas or cranial irradiation.

\section{Pathology}

Gliosarcomas are very similar to glioblastomas but with an added sarcomatous component. Although the tumour comprises of both glial and mesenchymal elements, there is evidence that both components arise from a solitary precursor cell.

They are almost invariably found in the cerebral hemispheres, and there may be slight predilection towards the temporal lobes. 


\section{Appearance}

These tumours vary in appearance depending on the relative amounts of sarcomatous tissue and astrocytic tissue. When the former is dominant, then these lesions appear similar to metastases; well circumscribed and firm. When the astrocytic component is abundant, then appearances are identical to a glioblastoma

Microscopically, these tumours show both an astrocytic component identical to glioblastomas and a sarcomatous component which is varied in differentiation, typically with epithelial components forming squamous tissue or gland-like structures Differentiation into many other types of tissue is also occasionally encountered (cartilage, bone, adipose, muscle).

\section{Radiographic features}

Gliosarcomas can be very similar to glioblastomas in appearance. They are usually broad-based peripherally located lesions with possible direct dural invasion or only reactive dural thickening (dural tail).

\section{CT}

Gliosarcomas may be seen on CT as a sharply defined (often due to sarcomatous component), round or lobulated, hyperdense solid mass. They can have relatively homogeneous contrast enhancement and peritumoral oedema.

\section{MRI}

Reported signal characteristics include:

T1: heterogeneous and hypointense mass

> T2: heterogeneous signal due hemorrhagic and necrotic components

$>\mathrm{T1C}+$ (Gadolinium): thick irregular and rim-like or ring enhancement

\section{MATERIALS AND METHODS}

This is a retrospective study conducted during the period between 2012 and 2016. In this study, there were 15 cases of gliosarcoma of the brain and spine had been selected to satisfy this study of gliosarcoma.

Patients were imaged with either one or two imaging modalities, Computed Tomography and Magnetic Resonance Imaging. Nine patients oc this sample were scannned using a 64 multi slice CT scan using the protocol of the brain imaging in which the patient was scan in supine position with the head of the patient is centered and fixed in the Gantry of CT Scan machine. Brain scan was done under selected exposure factors with slice thickness of $8 \mathrm{~mm}$ and intravenous Low Osmolar Water soluble Contrast Media (LOCM) was injected to the patients. Interpretation of the brain scan was performed by consultant radiologists to conform the diagnosis. The remaining six patients of this study were imaged with 1.5 Tesla Magnetic Resonance Imaging (MRI) by using the protocol of imaging of the head by MRI in which the following parameters of imaging were selected; $10 \mathrm{~mm}$ slice thickness of the brain, T1, T1 with contrast, T2 and FLAIR weighted images were done and the contrast media that used is intravenous Gadolinium $(0.1 \mathrm{ml} / \mathrm{kg})$ according to the weight of the patient.

The collected data in the study was analysed by using the SPSS software programme. The study contains both qualitative and quantitative variable. The quantitative were described using numbers, percentage and tables.

\section{RESULT}

The study included 15 patients that nine patients $(60 \%)$ were males while six patients $(40 \%)$ were female. Their age ranged from 12 to 73 years old. They had been referred for CT scan or MRI examination of the brain. (Table 1)

Table 1: Demographic data of the patient $(n=15)$

\begin{tabular}{|c|c|c|}
\hline $\begin{array}{c}\text { Patient } \\
\text { Gender }\end{array}$ & $\begin{array}{c}\text { No. of } \\
\text { respondents }\end{array}$ & Percentage (\%) \\
\hline Male & 9 & 60 \\
\hline Female & 6 & 40 \\
\hline
\end{tabular}

About 12 cases $(80 \%)$ of the gliosarcoma showed solid and cystic content, while 2 cases showed predominant solid content and one obviously cystic (Table 2) 
Table 2: Tumor content $(n=15)$

\begin{tabular}{|c|c|c|}
\hline Tumor Content & Frequency & Percentage(\%) \\
\hline Solid + Cystic & 12 & 80.0 \\
\hline Predominant Solid & 2 & 13.3 \\
\hline Obviously Cystic & 1 & 6.7 \\
\hline
\end{tabular}

Eleven of the gliosarcoma cases (73.3 \%) showed hypo intense in the T1 weighted image of MRI and two cases $(13.3 \%)$ showed hyper and iso intensity. one case showed iso intensity and one showed hypo and iso intensity. (Table 3)

Table 3: Tumor appearance in the MRI T1 weighted image $(n=15)$

\begin{tabular}{|c|c|c|}
\hline Tumor Appearance & Frequency & Percentage(\%) \\
\hline Hypo intense & 11 & 73.3 \\
\hline Hyper intense + iso intense & 2 & 13.3 \\
\hline Iso intense & 1 & 6.7 \\
\hline Hypo intense + iso intense & 1 & 6.7 \\
\hline
\end{tabular}

In the T2 weighted MRI image of gliosarcoma six cases showed hyper intensity, eight cases showed hyper intensity and is intensity, and only one case showed mixed intensity. (Table 4)

Table 4: Tumor appearance in the MRI T2 weighted image $(n=15)$

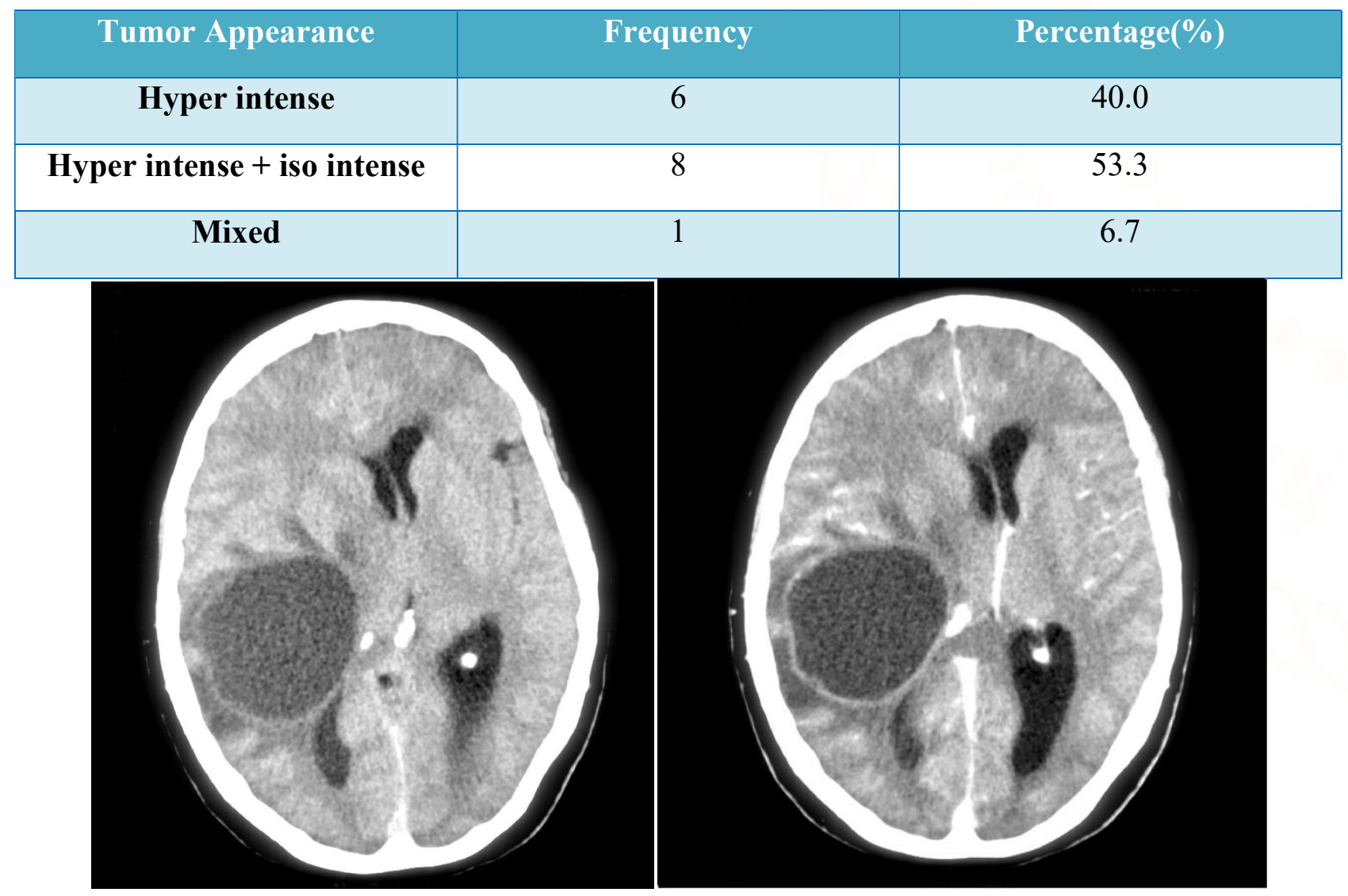


Figure 1: Selected pre and post-contrast images of brain showing a large cystic and well-defined lesion located in the right temporoparietal junction. Its content is homogeneously hypoattenuating and there is enhancement of the entire wall. The tumour promotes an expressive local mass effect characterised sulci effacement, perilesional oedema, right lateral ventricle partial collapse and midline shift to the left.
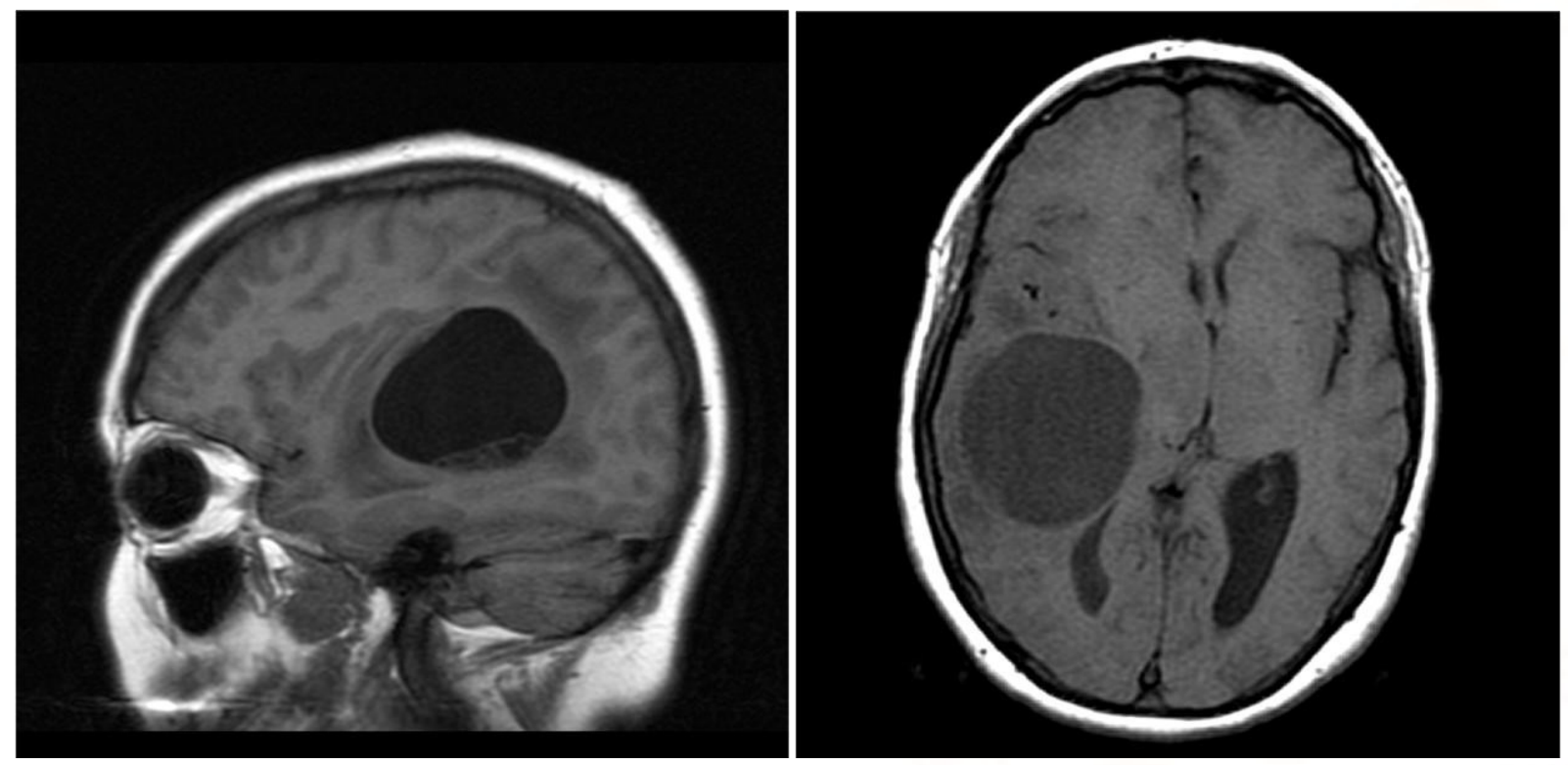

Figure 2: MRI T1 weighted image on sagital and axial section showing a large cystic and well-defined lesion located in the right temporoparietal junction. Its content is homogeneously hypo attenuating.

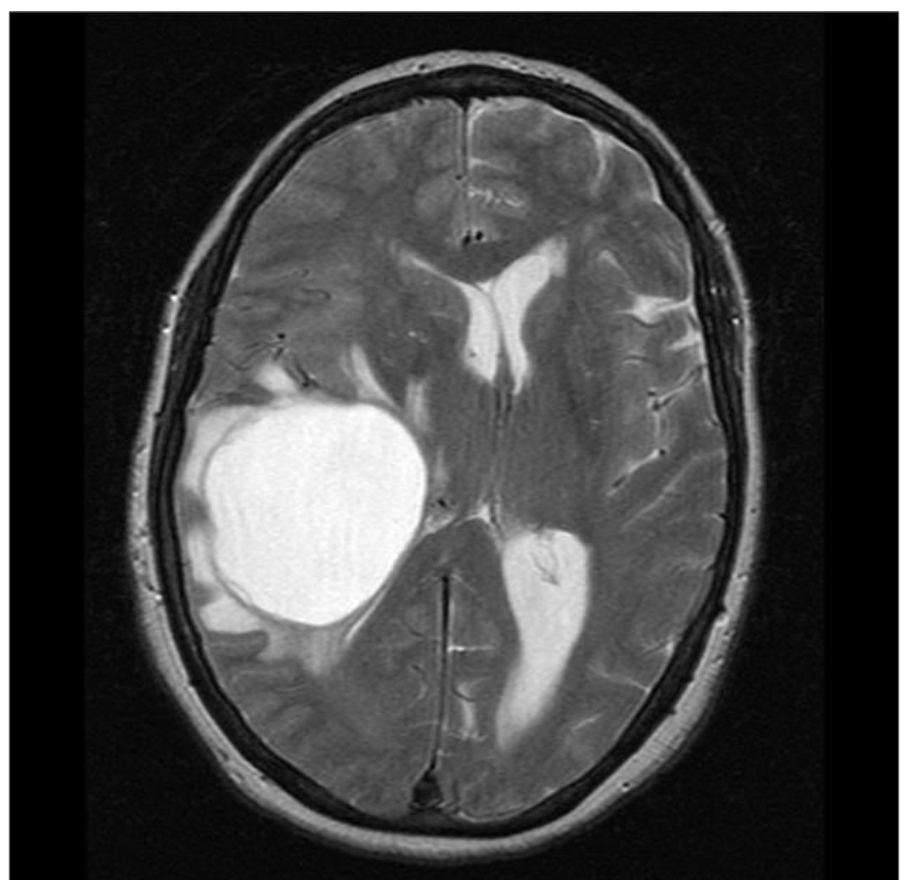

Figure 3: MRI T2 weighted image on axial section showing a large cystic and well-defined lesion located in the right temporoparietal junction. Its content is homogeneously hyper attenuating.

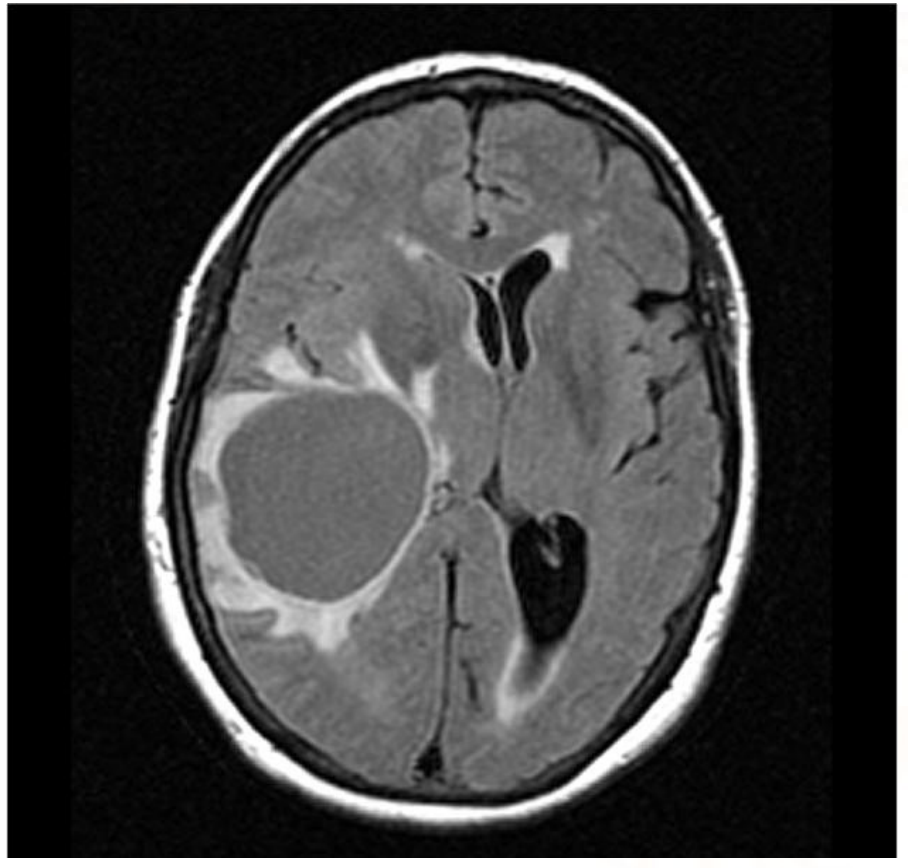

Figure 4: MRI FLAIR image on axial section showing a large cystic and well-defined lesion located in the right temporoparietal junction. Its content is homogeneously hypo attenuating. 


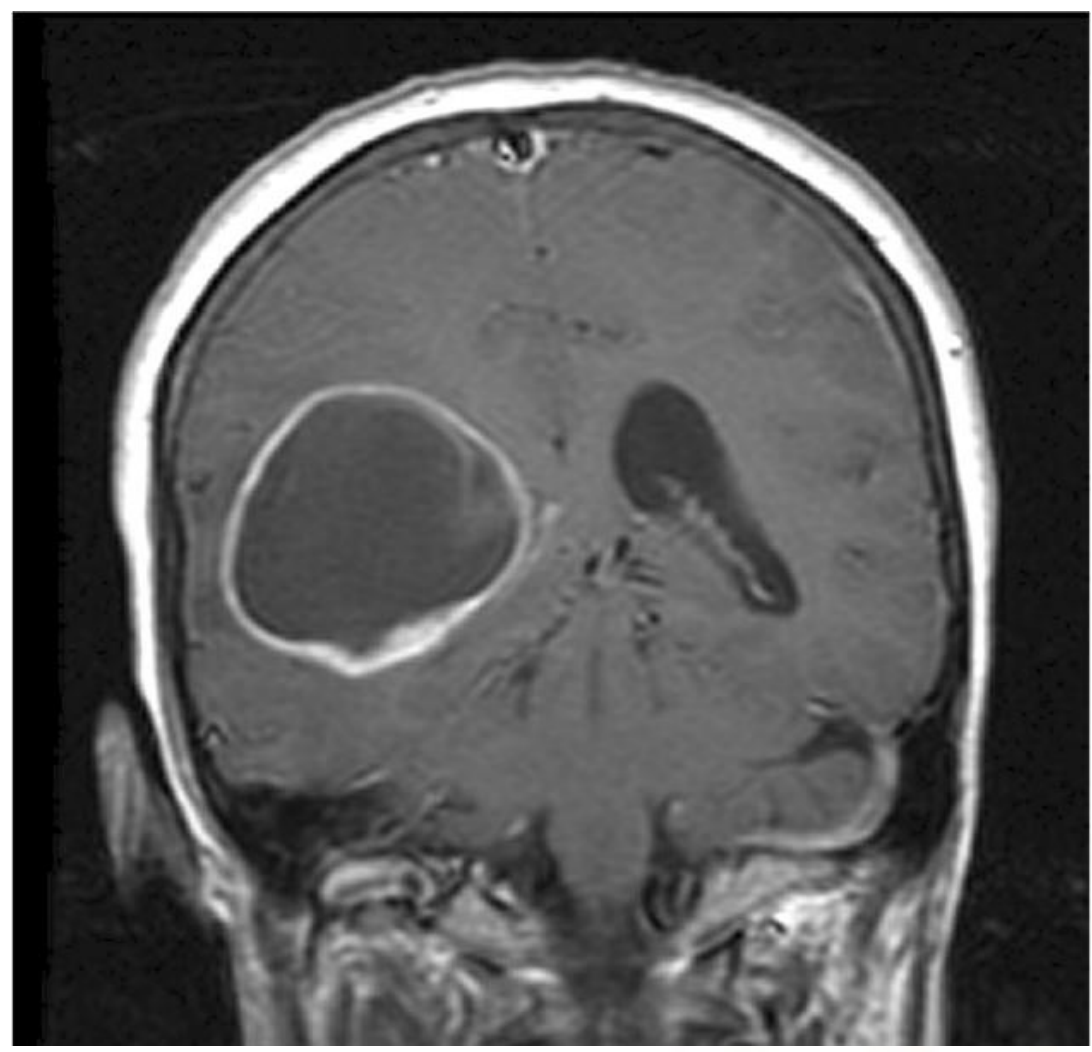

Figure 5: MRI T 1 weighted Contrast enhanced image on coronal section showing a large cystic and well-defined lesion located in the right temporoparietal junction. Its content is homogeneously hypo attenuating with contrast enhancement in the boundary.

\section{DISCUSSION}

Patients were imaged with either one or two imaging modalities, Computed Tomography and Magnetic Resonance Imaging . All tumors were visualized with contrast-enhanced MRI which proved most sensitive. Gadolinium di-ethylene triamine penta-acetic acid caused bright enhancement of tumors in a distribution that consistently corresponded to areas of pathologically proved tumor infiltration. Gross and microscopic autopsy findings correlated better with MRI than with CT which tended to produce poorer resolution and under represent the size of viable tumor. Gadolinium-enhanced MRI is more accurate than unenhanced MRI, unenhanced CT, or enhanced $\mathrm{CT}$ in defining the histologic margins of tumors.

Gliosarcomas were seen on CT as a sharply defined, round or lobulated, hypodense solid mass. They can have relatively homogeneous contrast enhancement and peritumoral oedema
Fourteen gliosarcomas were single. One case involved multifocal lesions. The multifocal masses were located in the hypothalamus and temporal and occipital lobes on the left side.

Tumors ranged from 2.9 to $12.0 \mathrm{~cm}$ in greatest diameter, with a mean of $5.3 \mathrm{~cm}$. Thirteen lesions had at least one dimension greater than $4 \mathrm{~cm}$. Only one tumor was smaller than $3 \mathrm{~cm}$.

Eleven gliosarcomas had both solid and cystic components. Two had predominantly solid components, and one tumor in the right cerebellar hemisphere had obvious cystic and/or necrotic areas. All 15 tumors appeared to be relatively well demarcated from the surrounding normal or edematous brain parenchyma. Contrast-enhanced T1weighted images depicted the boundaries of the tumors most clearly. (Figure 5)

Thirteen gliosarcomas were associated with vasogenic edema, as determined by reviewing the extent of peritumoral hyperintensity on T2-weighted images.(Figure 3) Ten tumors had moderate-toextensive adjacent edema, three tumors showed mild surrounding edema, and two had no apparent peritumoral edema. 
On T1-weighted images, 10 gliosarcomas were hypointense relative to adjacent normal white matter. (Figure 2). Two were both hyperintense and isointense, one was isointense, and one was hypointense and isointense. One lesion had a mixed appearance with areas of hypointensity, isointensity, and hyperintensity. In addition, three tumors showed scattered hyperintensity due to intratumoral hemorrhage ).

On T2-weighted images, six tumors were hyperintense relative to the surrounding normal brain parenchyma. Eight showed areas of hyperintensity and isointensity, and one had mixed high, low, and parenchyma-like signal intensity.

On FLAIR images, seven lesions were hyperintense to normal white matter. One tumor was isointense to hyperintense, and one was isointense.

Apart from the one gliosarcoma with moderate heterogeneous enhancement, all tumors showed strong heterogeneous enhancement. Thick walls with irregular, strongly enhancing rim and ring-like enhancement were observed in 13 (87\%) lesions. Seven $(47 \%)$ showed intratumoral strip and paliform enhancement. Four tumors demonstrated nodular enhancement on their walls. In one case, strong nodular enhancement was accompanied by small pieces of necrosis in the tumor. Images in three cases showed dural tails after the administration of contrast medium.

\section{CONCLUSION}

Gliosarcoma are more common in male than female. The tumor were mostly involving the parietal lobes with mass effect depending upon the grade.Gadolinium-enhanced MRI is more accurate than unenhanced MRI, unenhanced CT, or enhanced $\mathrm{CT}$ in defining the histologic margins of tumors.

\section{REFERENCE}

1) Stroebe H. Uber Entstehung und Bau der Gehirngliome. Beitr Pathol Anat Allg Pathol. 1895;18:405-86.

2) Feigen IH, Gross SW. Sarcoma arising in glioblastoma of the brain. Am J Pathol. 1955;31:633-53.

3) Morantz RA, Feigen I, Ransohoff J. Clinical and pathological study of 24 cases of gliosarcoma. J Neurosurg. 1976;45:398-408.
4) Meis JM, Martz Kl, Nelson JS. Mixed glioblastoma multiforme and sarcoma. A clinicopathologic study of 26 Radiation Therapy Oncology Group cases. Cancer. 1991;67:2342-9.

5) Perry JR, Ang LC, Bilbao JM, Muller PJ. Clinicopathologic features of prima- ry and postradiation cerebral gliosarcoma. Cancer. 1995;75:2910-18.

6) Damodaran O, van Heerden J, Nowak AK, Bynevelt M, McDonald K, Marsh J. Clinical management and survival outcomes of gliosarcomas in the era of multimodality therapy. J Clin Neurosci. 2014;21:478-81.

7) Lutterbach J, Guttenberger R, Pagenstecher A. Gliosarcoma: a clinical study. Radiother Oncol. 2001;61:57-64.

8) Zhang BY, Chen H, Geng DY, Yin B, Li YX, Zhong $\mathrm{P}$, et al. Computed tomography and magnetic resonance features of gliosarcoma: a study of 54 cases. J Comput Assist Tomogr. 2011;35:667-73.

9) Khalid AS, Mohamed O, Mohammed EM, Hassan S, Khalid H, Hamid M. Secondary gliosarcoma after the treatment of primary glioblastoma multiforme. North American Journal of Medical Sciences. 2011;3:527.

10) Schrantz JL, Araoz CA. Radiation induced meningeal fibro- sarcoma. Arch Pathol. 1972;93:26-33.

11) Han SJ, Yang I, Tihan T, Chang SM, Parsa AT. Secondary gliosarcoma: a review of clinical features and pathological diagnosis. J Neurosurg. 2010;112:26-32.

12) Kozak KR, Mahadevan A, Moody JS. Adult gliosarcoma: epidemiology, natural history, and factors associated with outcome. Neuro Oncol. 2009;11:183-91.

13) Salvati M, Caroli E, Raco A, Giangaspero F, Delfini R, Ferrante L. Gliosarcomas: analysis of 11 cases do two subtypes exist? J Neurooncol. 2005;74:59-63. 